\title{
Homogeneity of 67P/Churyumov-Gerasimenko as seen by CONSERT: implication on composition and formation
}

\author{
A. Herique ${ }^{1}$, W. Kofman ${ }^{1,2}$, S. Zine ${ }^{1}$, J. Blum ${ }^{3}$, J.-B. Vincent ${ }^{4}$, and V. Ciarletti ${ }^{5}$ \\ ${ }^{1}$ Univ. Grenoble Alpes, CNRS, CNES, IPAG, 38000 Grenoble, France \\ e-mail: alain.herique@univ-grenoble-alpes.fr \\ 2 Space Research Centre, PAN, Warsaw, Poland \\ ${ }^{3}$ Institut für Geophysik und extraterrestrische Physik, TU Braunschweig, Germany \\ ${ }^{4}$ DLR Institute of Planetary Research, Berlin, Germany \\ 5 LATMOS/IPSL, UVSQ, UPMC, CNRS, Guyancourt, France
}

Received 14 December 2018 / Accepted 19 April 2019

\begin{abstract}
Context. After the landing of Philae, CONSERT probed the nucleus of 67P/Churyumov-Gerasimenko (67P) and observed no heterogeneities at metric scale within the probed part of the small lobe of 67P. Further studies have then quantified the observed homogeneity in terms of maximum permittivity contrast versus the typical size of heterogeneities.

Aims. The aim of this article is to interpret the sensitivity limits of CONSERT measurements in terms of composition, and to provide constraints on the maximum variability in composition, porosity, and local dust-to-ice ratio.

Methods. The sensitivity of CONSERT measurements to local variations in density, dust-to-ice ratio, and composition was analyzed using permittivity modeling of mixtures.

Results. We interpret the maximum detectable heterogeneity size and contrast in terms of composition and porosity of the nucleus. The sensitivity to porosity is \pm 10 percent points for heterogeneities with a characteristic length scale of a few meters; the sensitivity to local variations in the composition is limited.

Conclusions. In terms of accretion, our results are compatible only with scenarios generating porosity heterogeneities at scales lower than one meter, or with porosity variations smaller than \pm 10 percent points. This is clearly compatible with an accretion model of a gentle gravitational collapse of a pebble cloud.
\end{abstract}

Key words. comets: individual: 67P/Churyumov-Gerasimenko - planets and satellites: formation - techniques: radar astronomy space vehicles: instruments

\section{Introduction}

After the landing of Philae, the Comet Nucleus Sounding Experiment by Radiowave Transmission (CONSERT) on board Rosetta probed the nucleus of 67P/Churyumov-Gerasimenko (67P) and observed no heterogeneities at metric scale within the fathomed part of the small lobe of the comet (Kofman et al. 2015). The observed homogeneity has later been quantified in term of maximum permittivity contrast versus typical size (Ciarletti et al. 2017). The aim of this paper is to interpret this limit in sensitivity of the measurements in terms of composition, and to provide constraints on the maximum variability in composition, porosity, and local dust-to-ice mass ratio (D/I). These constraints can then be compared to comet accretion scenarios and surface features observed by the Optical, Spectroscopic, and Infrared Remote Imaging System (OSIRIS) camera.

\section{Are clods the building blocks and evidence of a primordial or secondary accretion?}

Cometary nuclei represent the most primitive matter in the solar system in an almost pristine morphology (Schwartz et al. 2018). Thus, data gathered by the CONSERT experiment during the Rosetta and Philae mission may help to constrain planetesimal-formation models if these predict sufficiently highdensity contrast on sufficiently large length scales.

The two most relevant competing formation models for kilometer-sized planetesimals are described below.

- Model A: gravitational collapse of a pebble cloud. Primordial (sub-) micrometer-sized dust and ice grains coagulate into millimeter- to centimeter-sized aggregates (pebbles), which can no longer stick to each other because of the bouncing barrier (Zsom et al. 2010; Lorek et al. 2018). These pebbles are locally concentrated by one of many possible processes, for example, by turbulent concentration, sedimentation in a quiescent nebula, or Kelvin-Helmholtz instability (see Johansen et al. 2014 for an overview), until the streaming instability is triggered, which further enhances the local concentration of pebbles (Youdin \& Goodman 2005). If the conditions for pebble size and nebula metallicity are met (Yang et al. 2017), a gravitational collapse can occur that directly forms planetesimals with sizes of up to $\sim 1000 \mathrm{~km}$ (Johansen et al. 2007).

- Model B: hierarchical collisional growth. After the pebbles have coagulated out of the primordial dust and ice particles, they overcome the bouncing barrier (Windmark et al. 2012; Garaud et al. 2013) and continue to grow by mass transfer, for instance (see Güttler et al. 2010 for a list of growth processes). Weidenschilling (1997) derived the foundations for the 
hierarchical growth model of cometesimals, and Davidsson et al. (2016) applied this model to comet 67P and Rosetta findings. Because of their aerodynamic properties, the growing bodies experience mutual collisions primarily with bodies that typically are a factor of several smaller than themselves. The mean collision speeds first increase until they reach a maximum of $\sim 30 \mathrm{~m} \mathrm{~s}^{-1}$ for meter-sized bodies and then decrease again.

The collisional properties of dust aggregates have been studied by Bukhari Syed et al. (2017) and can be applied to the two planetesimal-formation models to derive the expected amount of inhomogeneity, which can then be compared to the results of CONSERT.

Homogeneity and characteristic length scales of planetesimals formed by model A: Wahlberg Johansen et al. (2017) have applied the dust-aggregate collision model by Bukhari Syed et al. (2017) to collapsing pebble clouds and found that for bodies $\lesssim 10 \mathrm{~km}$ in size, all pebbles survive intact. Moreover, the amount of lithostatic compression inside such small bodies is so low that plastic deformation of the pebbles is not expected either (Blum 2018). As the pebble sizes are constrained by the efficiency of the streaming instability to radii $\lesssim 3 \mathrm{~cm}$ at 30 AU (Blum et al. 2017), model A does not predict a measurable density contrast at the meter-scale or larger, except for late-stage impact processes and evolutionary changes, which might affect only the near-surface regions.

Homogeneity and characteristic length scales of planetesimals formed by model B: through the increasing collision velocity with increasing size for bodies smaller than meters (Weidenschilling 1997; Davidsson et al. 2016), all pebbles will be collisionally destroyed. Davidsson et al. (2016) argued that comets formed by model B contain abundant meter-sized bodies because they were formed at the highest collision speeds and are thus the hardest to destroy. Moreover, comet 67P should also contain cavities with sizes $\sim 1-100 \mathrm{~m}$ in its interior. However, applying the dust-aggregate collision model by Bukhari Syed et al. (2017) to the impacts predicted by model B shows that all projectiles $>1 \mathrm{~m}$ in size are completely collisionally destroyed. Moreover, even the gentlest collisions in the model by Davidsson et al. (2016), that is, between $500 \mathrm{~m}$ targets and $200 \mathrm{~m}$ projectiles at $0.2 \mathrm{~m} \mathrm{~s}^{-1}$ impact speed, would lead to a catastrophic fragmentation of the target according to the impact model by Bukhari Syed et al. (2017). Thus, the growing bodies must be collisional rubble piles, which should contain measurable density contrasts in their interiors. Following the model by Bukhari Syed et al. (2017), the largest intact remnant of the collisional rubble pile should contain $\sim 1-10 \%$ of the target mass, with smaller fragments following a power-law in size-frequency distribution. It should also be noted that the impact pressure in the model by Weidenschilling (1997) is never sufficiently high to structurally destroy the fragments that are produced in previous impacts.

To summarize, the two competing planetesimal-formation models predict very different internal morphologies for cometary nuclei, which might be compared with the results from CONSERT and from the optical remote sensing of comet surfaces.

\section{Compositional homogeneity of $67 \mathrm{P}$ as seen by CONSERT}

\subsection{CONSERT experiment and the internal structure of the nucleus}

CONSERT was a radar on board both the Rosetta spacecraft and its lander Philae, designed to probe the nucleus of $67 \mathrm{P}$ in transmission and to address the question of its heterogeneity or homogeneity (Kofman et al. 1998, 2007). After the landing of Philae on the surface of comet 67P at 17:31 UTC on 12 November 2014, CONSERT operated for $\sim 9 \mathrm{~h}$; the radio waves traveled through the nucleus with a free space wavelength of $\sim 3 \mathrm{~m}$. Strong signals were detected for about 30 and $80 \mathrm{~min}$ at the beginning and end of the acquisition sequence, whereas the remaining signals were affected by a low signal-tonoise ratio $(\mathrm{S} / \mathrm{N})$. CONSERT probed a limited part of the small lobe of 67P, which corresponds to the southern border of the Hatmehit depression, around Abydos, with an expected penetration depth of up to $100 \mathrm{~m}$ and a propagation length of up to around $1 \mathrm{~km}$.

The first analysis of the CONSERT measurements (Kofman et al. 2015) showed that these strong signals propagated through parts of the nucleus and were as narrow as the calibration signals acquired on ground or during the Rosetta cruise. Therefore, there is no signature of volume- or surface-scattering effects in the signal form down to $-20 \mathrm{~dB}$ below the signal peak. If scattering were present, a long tail of decay in the signal should be visible (Boisson et al. 2011). The absence of this scattering indicates that the medium explored by the waves was rather homogeneous and/or that the dielectric contrast (difference) between potential inclusions inside the nucleus was low, at least at a scale comparable to the wavelength $(\sim 15 \mathrm{~cm}-15 \mathrm{~m})$. The real part of the permittivity is inferred from the propagation time and is equal to $1.27 \pm 0.05$

As a second step, the absence of scattering was quantified in terms of maximum size of the possible scatterers and of maximum deviation with respect to the average bulk permittivity, in order to assess the maximum size and contrast of the possible heterogeneities for which no signal spreading can be observed. This study was carried out using numerical simulations of electromagnetic wave propagation (Ciarletti et al. 2017). The results are listed below.

- CONSERT is not sensitive to any $1 \mathrm{~m}$ scale potential structures and can provide no constraint on the dielectric contrast of this type of heterogeneities. This limitation is mainly due to the high porosity and consequently to the low permittivity, which limits interaction between waves and material.

- If heterogeneities exist in the range 1-10 $\mathrm{m}$, they have to correspond to a dielectric contrast lower than $\Delta \varepsilon=\varepsilon_{\max }-$ $\varepsilon_{\min }=0.25$.

The question now arises about the meaning of these limitations in terms of deviation from the average composition and porosity. Answering this question first requires inferring information about the composition from the measured average permittivity.

\subsection{P: average composition}

The CONSERT measurements allowed determining the average permittivity of the comet. From this value, we can evaluate its internal composition and porosity. Applying Hashin-Shtrikman mixing formulas, the compositions and porosities that are compatible with the CONSERT-derived value of permittivity can be calculated (Kofman et al. 2015; Herique et al. 2016). The approach is explained in detail by Herique et al. (2016): the cometary material is modeled by a mixture of three constituents:

- an ice fraction that is dominated by hexagonal water ice plus possible fractions of $\mathrm{CO}, \mathrm{CO}_{2}$, and amorphous ice, with a mass fraction of $\mathrm{CO}_{2}$ and $\mathrm{CO}$ up to $15 \%$ each;

- a refractory fraction with different tested candidates consisting of minerals, organics, and meteoritic materials, the 


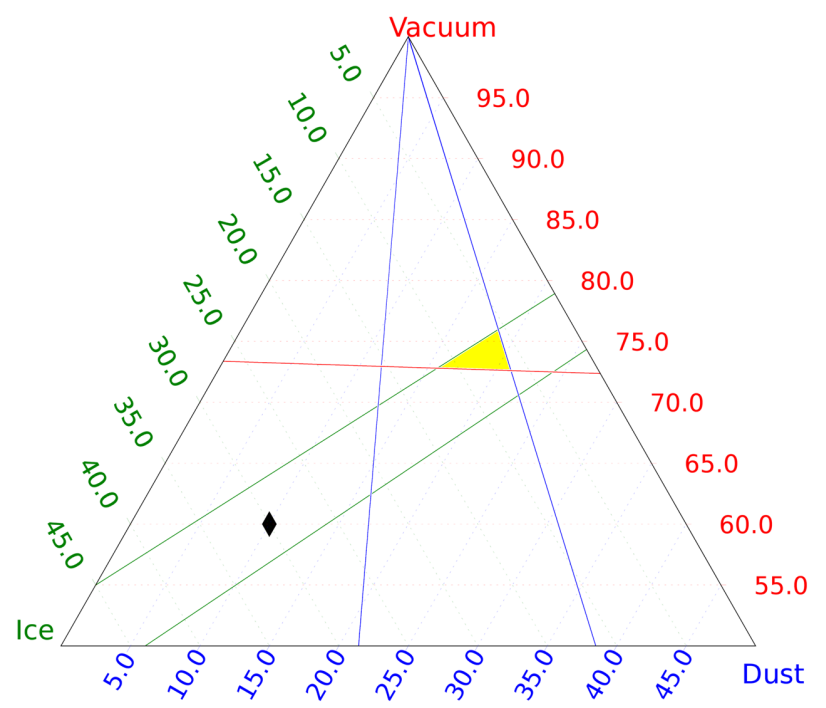

Fig. 1. Constraints from density (green), D/I (blue) and CONSERT (red), and possible domain of volume ratio (yellow triangle) for a mixture of $75 \%$ organics / $25 \%$ silicates in volume. Only the upper quarter of the ternary diagram is plotted (porosity higher than 50\%). The black diamond corresponds to a mixture of $30 \%$ ice, $10 \%$ dust, and $60 \%$ porosity.

permittivities of which were obtained from laboratory measurements (see review in Herique et al. 2016);

- vacuum (i.e., porosity).

We considered the mixture at a macroscopic scale: the topology of microscopic ice, mineral, and organics grains is not discussed. We have shown in the previous paragraph that any submetric heterogeneities are not visible to CONSERT.

These results can be presented in a ternary diagram, as in Fig. 1 (Kamoun et al. 2014): this allows us to take constraints given by other instruments into account, such as the measured density (green lines) and D/I (blue lines). The green line limits the possible domain by the average density of the comet $\left(533 \pm 6 \mathrm{~kg} \mathrm{~m}^{-3}\right)$, the blue line by the possible $\mathrm{D} / \mathrm{I}(2 \leq \mathrm{D} / \mathrm{I} \leq 6$, Rotundi et al. 2015), and the red line by the measurements of CONSERT. Both density and D/I constraints (green and blue lines) depend on assumed dust densities, which can be refined for each candidate. The yellow triangle in Fig. 1 is the only possible domain of volume fractions that is compatible with all these constraints for the considered dust composition, and it corresponds to a limited range of possible composition and porosity.

The test of different composition models based on a large set of minerals, organics, and meteoritic materials shows that only a solution with a large portion of organics is possible (see Herique et al. 2016 for details). Of all the end members we tested, the carbon with $\mathrm{Mg}$-silicate (16\% olivine, $9 \%$ pyroxene, and $75 \%$ carbon) and the UCAM-like pure refractory carbon (100\% carbon) are compliant with CONSERT measurements when the constraint on average density is taken into account. The carbonaceous chondrites are compatible with CONSERT measurements, but they are excluded on the basis of VIRTIS observations (Quirico et al. 2016).

The yellow triangle shows the possible volume-fraction domain for a dust fraction composed of $75 \%$ organics and $25 \%$ silicates in volume, which corresponds to $68 \%$ organics and $32 \%$ silicates in mass, with a density of $2300 \mathrm{~kg} \mathrm{~m}^{-3}$ and a permittivity of 2.6. Figure 1 shows that the CONSERT-compatible interior composition of the comet has an ice volume fraction

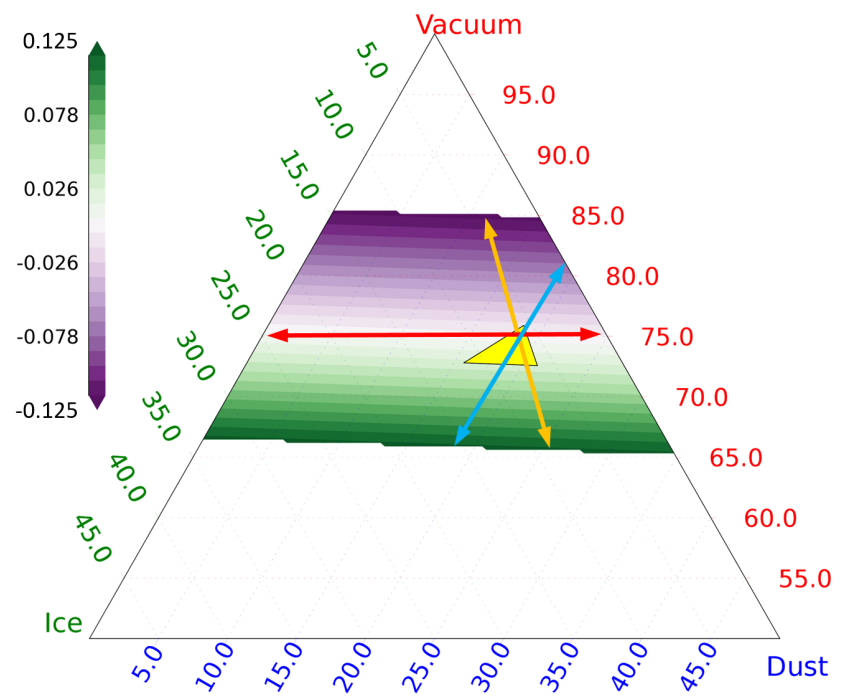

Fig. 2. Permittivity variations of the dust, ice, and vacuum mixture for corresponding variations in composition. The ice fraction correspond to a mixture of $73 \% \mathrm{H}_{2} \mathrm{O}$ ice (mass fraction), $14 \% \mathrm{CO}_{2}$ ice, and $15 \%$ $\mathrm{CO}$ ice with an effective permittivity equal to 2.7 . The dust fraction is a mixture of $68 \%$ of organics (mass fraction) and $32 \%$ silicates for an effective permittivity equal to 2.6. The orange arrow corresponds to a variation in the density and constant composition and D/I (case a), the red arrow to a variation in the $\mathrm{D} / \mathrm{I}$ and constant porosity and composition (case b), and the blue arrow to the same dust fraction, but desiccated or ice enriched.

in the interval $5-11 \%$ and a refractory volume fraction in the $16-21 \%$ interval with a porosity ranging from 73 to $76 \%$. We point out that this result corresponds to the average permittivity, considering global constraints on the average density and D/I.

\subsection{Composition variability}

We used a similar approach to analyze the comet homogeneity seen by CONSERT: our interpretation relies on the same dielectric mixing models, but here we focus on the influence of the local variability, setting aside the constraints on average permittivity, density, and D/I. It remains to be defined which constraints the permittivity contrast limit places on the variability of composition or porosity. To answer this question, we freely move outside the yellow triangle in Fig. 1, as long as the local variations, that is, the variations of permittivity of any subscale inclusions, cannot cause $\varepsilon$ to vary by more than $\Delta \varepsilon=0.25$ around the CONSERT average value of 1.27 . Meanwhile, the average values must still fit the average permittivity and densities.

Figure 2 shows the permittivity of the dust-ice-vacuum mixture assuming both ice and dust remain the same in terms of composition, while their volume fractions (including porosity) vary. The permittivity was calculated using the upper limit of the Hashin-Shtrikman formula, as done by Herique et al. (2016), for a mixture of ice with $\varepsilon_{\text {ice }}=2.7$ and dust with $\varepsilon_{\text {dust }}=2.6$ for different volume ratios and porosities. The color scale represents the allowed $\Delta \varepsilon=0.25$ permittivity variability around its mean value, that is, $\varepsilon \pm \frac{\Delta \varepsilon}{2}$ for the above-described median point within the yellow triangle, representing a given composition (and assuming the types of ice and refractory fraction do not vary). This permittivity variability results in a wide range of variations in terms of volume fraction: the possible resulting mixture variations can be located anywhere within a band of the ternary diagram, with the 
ice volume fraction within the $17-35 \%$ interval, the dust volume fraction within the $0-50 \%$ interval, and the porosity within 63-83\% interval. We would like to point out that these values remain compatible with an average permittivity of 1.27.

To better understand this result, we consider two different cases:

- Local density variation: in the first case, only the density varies locally, but the composition remains the same everywhere in the nucleus (same D/I and same composition of the ice and of the refractory material). In this case, we move in the ternary diagram along the line that contains the considered point within the yellow triangle and the upper vertex of the diagram (the latter corresponding to a porosity of $100 \%$ ). This case is marked by an orange arrow in Fig. 2. The range of the allowed permittivity variation limits the possible porosity variation to $65-85 \%$. The range of variation in porosity must remain within $\pm 10 \%$ points in order to induce no scattering.

- Local variation in the D/I: a second case consists of a variation in the local D/I ratio while the porosity and the composition of the ice and dust fractions remain constant. In this case, we move in the ternary diagram along the horizontal line represented by the red arrow in Fig. 2. Then the possible variations span the whole diagram: we can move from pure porous ice to pure dry dust. In other words, CONSERT shows no sensitivity to distinguishing ice from dust at this scale because both fractions are very comparable in terms of permittivities (2.7 and 2.6, respectively) and a change in the local D/I does not significantly affect the permittivity of the resulting mixture. The situation is entirely different for the average values: an additional constraint due to the average density allows distinguishing between ice and dust because the density contrasts between them are very high (1 and 2.3, respectively).

Many alternative scenarios can be envisaged around these two cases. For example, the blue arrow in Fig. 2 corresponds to a constant dust fraction that is desiccated or ice enriched, with a composition that varies from entirely desiccated dust to an ice volume fraction of up to $15 \%$. This corresponds to a porosity of about $68 \%$, which is very close the $65 \%$ porosity of case (a). For alternative models, the porosity variation is the driver at the first order, which corresponds to the limited sensitivity in terms of local D/I.

It is definitively more difficult to quantify the heterogeneities induced by a variation in composition of each phase: the domain to be explored is large, especially for dust, and the ternary diagram is no longer relevant for synthesizing results and limits. We only considered two straw-man models of heterogeneity: a variation in permittivity of the ice fraction only, with a constant porosity and a constant dust fraction, and the inverse case with a variation in dust fraction only. The goal is then more to try to establish some limits than to test established models.

- Sensitivity to the ice fraction composition: there is no sensitivity to the ice: a variation from pure $\mathrm{CO}$ or $\mathrm{CO}_{2}$ ice $(\varepsilon=1.4$ and 2.1 , respectively) to pure water ice ( $\varepsilon=3.1$ and up to 3.4 for amorphous ice) induces a variation in permittivity in the order of 0.07 , significantly lower than our $\Delta \varepsilon=0.25$ limit. This is fully expected, considering that the average ice fraction typically is in the order of $6 \%$ (lower than the limit of the porosity variation as quantified in case a).

- Sensitivity to the dust fraction composition: for the dust fraction, the sensitivity is slightly higher. When we consider a variation in composition of the refractory fraction alone, all things being equal, our $\Delta \varepsilon=0.25$ limit corresponds to a variation in refractory fraction from pure organics $(\varepsilon=1.9)$ to a mixture of silicates and organics $(\varepsilon=5.2)$, and up to $70 \%$ volume fraction of silicates $(\varepsilon=7.1)$ and $30 \%$ of organics $(\varepsilon=1.9)$. This volume ratio is just to give an order of magnitude to show that dust composition can vary locally from pure organics to rather silicate material. We stress that the average composition must fit the $75 \%$ organics and $25 \%$ silicates calculated by Herique et al. (2016).

Again, this local variation in composition is just a limit of sensitivity of our measurements. It is also supported by internal structure modeling.

\section{Discussion and conclusions}

CONSERT observed a homogeneous nucleus at the scale of a few meters and above. This homogeneity was quantified by limiting the maximum variability range of the local composition and porosity versus the size of the heterogeneities, while the average composition and porosity have to fit the values previously derived from CONSERT measurements (Herique et al. 2016). CONSERT was not sensitive to any heterogeneity with dimensions of one meter or smaller. Therefore, the following conclusion concerns scales larger than a few meters (typically $3 \mathrm{~m}$ ).

The sensitivity of our measurements to the local variation in composition is very limited, and it is impossible to establish constraints on the variation of the local D/I or on the ice fraction composition. This is quite the same for the limit of the dust fraction composition: the authorized local variations range from pure organics up to $\sim 70 \%$ silicates because our sensitivity for the average composition comes from the joint constraints on average permittivity and average density. We cannot distinguish between models that only correspond to variations in local composition. The sensitivity to porosity is clearly better: if any heterogeneity exists at a scale of a few meters in the fathomed part of the small lobe of 67P, this heterogeneity has to correspond to a local variation in porosity lower than \pm 10 percent points (total range from $65 \%$ porosity to $85 \%$ ). This is the main conclusion of our analysis.

In terms of accretion, our conclusions are compatible only with scenarios that generate porosity heterogeneities at a scale smaller than one meter, or with porosity variations smaller than \pm 10 points. The former constraint is clearly compatible with model A (gentle gravitational collapse of a pebble cloud; see Sect. 2), which does not exhibit heterogeneities on size scales exceeding the pebble size, that is, a few centimeters at most. The predicted porosity of 73-78\% of planetesimals formed by model A (Blum et al. 2017) is also in full agreement with the results obtained with CONSERT. Because planetesimal matter consisting of pebbles is weak (Krivov et al. 2018), reaccumulation after a catastrophic collision would not result in a rubble-pile body, but would be structurally identical to the parent body before the collision. In model B, we have to distinguish between the original planetesimal and the body resulting from possible post-formation catastrophic collisions. As pointed out by Blum (2018), the expected porosity of a planetesimal formed by model B is $\sim 60 \%$, slightly below the lower limit allowed by the CONSERT measurements. Subsequent catastrophic collisions will produce fragments with a power-law size-frequency distribution, which will be gravitationally reaccumulated. If the collisional strength of these fragments is large enough to survive the gravitational reaccumulation, the forming body will be a rubble pile, whose constituents (fragments with porosity $\sim 60 \%$ ) and void space (with porosity $100 \%$ ) should exhibit a high contrast in permittivity. This disagrees with the findings by CONSERT, unless the largest fragment is smaller than a few meters. When the fragments are destroyed upon reaccumulation, 
we again expect a rather homogeneous body with porosity on the order of $60 \%$, which also disagrees with CONSERT data. Thus, we conclude that model A seems to be more likely than model B.

Finally, this result can be compared to optical observations of the surface of 67P. The first observations of the surface by OSIRIS after arrival at the comet in August 2014 revealed peculiar morphologies on several steep slopes, colloquially described as "goosebumps" (Sierks et al. 2015). They appeared as circular, possibly spherical, patterns on the walls of pits, with a size distribution that strongly peaked at about $3 \mathrm{~m}$. Because that scale was comparable to the order of magnitude of primordial pebbles, a potential link was postulated. Further observations revealed, however, that this interpretation is unlikely. Polygonal features of similar sizes are found all over the nucleus (Thomas et al. 2015), and their characteristics are well within the expected range of parameters for desiccation or thermal contraction polygons, as observed on many other bodies (Auger et al. 2018). In addition, the more rounded polygons are found in highly active areas, where jet-like features accelerated through the cracks will eventually erode the polygons and lead to the observed texture (Vincent et al. 2016). Overall, morphological and thermal model tend to strongly favor an evolutionary process, and it is unlikely that goosebumps are the expression of primordial pebbles. This interpretation of a non-primordial origin agrees with CONSERT observations, and any alternative scenario considering a pristine origin of these structures must match our maximum porosity variation of \pm 10 points.

Acknowledgements. CONSERT was designed, built, and operated by IPAG, LATMOS, and MPS, and was financially supported by CNES, CNRS, UGA, DLR, and MPS. Rosetta is an ESA mission with contributions from its Member States and NASA. Philae is provided by a consortium led by DLR, MPS, CNES, and ASI.

\section{References}

Auger, A.-T., Groussin, O., Jorda, L., et al. 2018, Icarus, 301, 173 Blum, J. 2018, Space Sci. Rev., 214, 52

Blum, J., Gundlach, B., Krause, M., et al. 2017, MNRAS, 469, S755

Boisson, J., Heggy, E., Clifford, S. M., et al. 2011, J. Geophys. Res., 116, E11003

Bukhari Syed, M., Blum, J., Wahlberg Jansson, K., \& Johansen, A. 2017, ApJ, 834,145

Ciarletti, V., Herique, A., Lasue, J., et al. 2017, MNRAS, 469, S805

Davidsson, B. J. R., Sierks, H., Güttler, C., et al. 2016, A\&A, 592, A63

Garaud, P., Meru, F., Galvagni, M., \& Olczak, C. 2013, ApJ, 764, 146

Güttler, C., Blum, J., Zsom, A., Ormel, C. W., \& Dullemond, C. P. 2010, A\&A, 513, A56

Herique, A., Kofman, W., Beck, P., et al. 2016, MNRAS, 462, S516

Johansen, A., Oishi, J. S., Mac Low, M.-M., et al. 2007, Nature, 448, 1022

Johansen, A., Blum, J., Tanaka, H., et al. 2014, in Protostars and Planets VI, eds.

H. Beuther, R. S. Klessen, C. P. Dullemond, \& T. K. Henning (Tucson, AZ: University of Arizona Press), 547

Kamoun, P., Lamy, P. L., Toth, I., \& Herique, A. 2014, A\&A, 568, A21

Kofman, W., Barbin, Y., Klinger, J., et al. 1998, Adv. Space Res., 21, 1589

Kofman, W., Herique, A., Goutail, J.-P., et al. 2007, Space Sci. Rev., 128, 413

Kofman, W., Herique, A., Barbin, Y., et al. 2015, Science, 349, aab0639

Krivov, A. V., Ide, A., Löhne, T., Johansen, A., \& Blum, J. 2018, MNRAS, 474, 2564

Lorek, S., Lacerda, P., \& Blum, J. 2018, A\&A, 611, A18

Quirico, E., Moroz, L. V., Schmitt, B., et al. 2016, Icarus, 272, 32

Rotundi, A., Sierks, H., Della Corte, V., et al. 2015, Science, 347, aaa3905

Schwartz, S. R., Michel, P., Jutzi, M., et al. 2018, Nat. Astron., 2, 379

Sierks, H., Barbieri, C., Lamy, P. L., et al. 2015, Science, 347, aaa1044

Thomas, N., Sierks, H., Barbieri, C., et al. 2015, Science, 347, aaa0440

Vincent, J.-B., Oklay, N., Pajola, M., et al. 2016, A\&A, 587, A14

Wahlberg Jansson, K., Johansen, A., Bukhari Syed, M., \& Blum, J. 2017, ApJ, 835,109

Weidenschilling, S. J. 1997, Icarus, 127, 290

Windmark, F., Birnstiel, T., Güttler, C., et al. 2012, A\&A, 540, A73

Yang, C.-C., Johansen, A., \& Carrera, D. 2017, A\&A, 606, A80

Youdin, A. N., \& Goodman, J. 2005, ApJ, 620, 459

Zsom, A., Ormel, C. W., Güttler, C., Blum, J., \& Dullemond, C. P. 2010, A\&A, 513, A57 